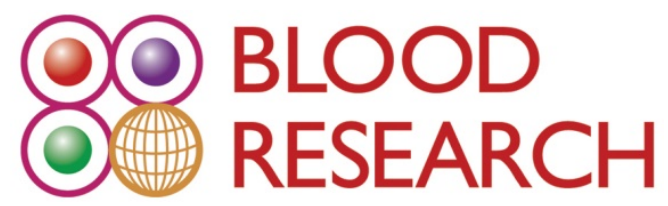

\title{
Convalescent plasma in COVID-19: renewed focus on the timing and effectiveness of an old therapy
}

Hebat- Allah Hasan Nashaat, Maha Anany, Fadia Moustafa Attia

Citation: Nashaat HAH, Anany M, Attia FM. Convalescent plasma in COVID-19: renewed focus on the timing and effectiveness of an old therapy. Blood Res 2022. [Epub ahead of print]

Publisher's disclaimer: This manuscript has completed peer review and has been accepted for publication. We are distributing the authors' version of manuscript prior to technical and English editing, typesetting and proof reading. A final version approved by the authors' will follow in a regular issue of BLOOD RESEARCH. All legal disclaimers that apply to BLOOD RESEARCH also pertain to this production process. 
Convalescent plasma in COVID-19: renewed focus on the timing and effectiveness of an old therapy

Hebat- Allah Hasan Nashaat, Maha Anany, Fadia Moustafa Attia

Clinical and Chemical Pathology, Faculty of Medicine, Suez Canal University, Ismailia, Egypt

Correspondence to: Heba-t Allah H. Nashaat, M.D., Ph.D.

Clinical and chemical Pathology, Faculty of Medicine, Suez Canal University, El Sheikh Zayed, Ismailia, Egypt

E-mail: hebaallah.hassan@med.suez.edu.eg 


\begin{abstract}
Severe acute respiratory syndrome coronavirus 2 (SARS-CoV-2) is responsible for the coronavirus disease 2019 (COVID-19) pandemic that has strained health care systems worldwide and resulted in high mortality. The current COVID-19 treatment is based on supportive and symptomatic care. Therefore, convalescent plasma (CP), which provides passive immunization against many infectious diseases, has been studied for COVID-19 management. To date, a large number of randomized and non-randomized clinical trials as well as many systematic reviews have revealed conflicting results. This article summarizes the basic principles of passive immunization, particularly addressing $\mathrm{CP}$ in COVID-19. It also evaluates the effectiveness of CP as a therapy in patients with COVID-19, clinical trial reports and systematic reviews, regulatory considerations and different protocols that are authorized in different countries to use it safely and effectively. An advanced search was carried out in major databases (PubMed, Cochrane Library, and MEDLINE) and Google Scholar using the following key words: SARS-CoV-2, COVID-19, convalescent plasma, and the applied query was "convalescent plasma" AND "COVID-19 OR SARS-CoV-2". The results were filtered and duplicate data were removed. Collective evidence indicates that two cardinal players determine the effectiveness of $\mathrm{CP}$ use, time of infusion, and quality of $\mathrm{CP}$. Early administration of $\mathrm{CP}$ with high neutralizing anti- anti-spike IgG titer is hypothesized to be effective in improving clinical outcome, prevent progression, decrease the length of hospital stay, and reduce mortality. However, more reliable, high quality, well-controlled, double-blinded, randomized, international and multicenter collaborative trials are still needed.
\end{abstract}

Key words: Convalescent plasma, COVID-19, SARS-CoV-2, Neutralizing antibodies 


\section{INTRODUCTION}

Severe acute respiratory syndrome coronavirus 2 (SARS-CoV-2), the etiological agent responsible for the coronavirus disease 2019 (COVID-19) pandemic, was first discovered in Wuhan (China) in December 2019. COVID-19 has a high transmission rate and 20.1\% and 25.9\% of patients develop acute respiratory distress syndrome and are admitted in the intensive care unit (ICU) for pneumonia, respectively. In addition, it has a mortality rate of approximately $6.84 \%[1,2]$. Currently, COVID-19 management is based on supportive care, such as the use of antipyretics, low-dose systematic corticosteroids, and anticoagulants; $\mathrm{O}_{2}$ therapy and invasive and non-invasive ventilation; and fluid management [3].

SARS-CoV-2 is a novel virus; therefore, no proven effective specific antiviral therapies are available yet. Notably, if vaccines or specific therapies are developed, the urgent need for large-scale production and distribution could be time-consuming. Hence, a long-standing classic adaptive immunotherapy technique that uses convalescent plasma (CP) has been applied to prevent and treat many infectious diseases over the years and can be used for COVID-19 management [4]. CP [passive polyclonal antibodies (Abs)] provides immediate immunity and can either be transfused to patients fighting an infection or used to manufacture immune globulin concentrates (plasma-derived medicinal products).

\section{Role of CP in patients with COVID-19}

CP could benefit patients with COVID-19 through its antiviral and immunomodulatory effects (Fig. 1).

\subsection{Antiviral mechanisms}

$\mathrm{CP}$ has neutralizing Abs (NAbs), and its efficacy is associated with the concentration of these NAbs [5]. These NAbs attach to the SARS-CoV-2 spike 1 receptor-binding domain, S1-N-terminal domain, and S2 subunit, inhibit virus entry, and limit viral amplification [6]. In addition, CP could also 
exert its therapeutic effects through other Ab-mediated pathways, such as complement activation, antibody-dependent cellular cytotoxicity, and/or phagocytosis. Of note, recovered patients have variable NAb titers [7] owing to age, lymphocyte count, and C-reactive protein levels; approximately $30 \%$ of patients do not have high NAb titers. Importantly, other protective non-NAbs, such as immunoglobulin (Ig)G and IgM, that bind to the virus without affecting its capacity to replicate, might contribute to recovery [8].

In one study, IgG production against the nucleoprotein $(\mathrm{N})$ and seroconversion could be detected on days 4 and 14 after disease onset, respectively [9]. Another study recorded the highest IgM concentration on the $9^{\text {th }}$ day after onset, and class switching to IgG occurred in the $2^{\text {nd }}$ week [10].

\subsection{Immunomodulation}

Anti-inflammatory cytokines, clotting factors, natural Abs, defensins, pentraxins, and other undefined proteins obtained from donors may provide further benefits such as immunomodulation.

\subsubsection{Antigen-binding fragment $\left[F\left(a b^{\prime}\right)\right] 2$ mechanisms}

Critically ill patients with COVID-19 produce anti-cardiolipin IgA and anti- $\beta 2$-glycoprotein I IgA and IgG Abs [11]. CP may neutralize these autoantibodies and reduce the risk of patients suffering thrombotic events (i.e., antiphospholipid syndrome-like disease), especially in critically ill patients. In addition, some Abs inhibit complement cascade (i.e., C3a and $\mathrm{C} 5 \mathrm{a}$ ) and limit the formation of immune complexes. The literature shows that other studies have illustrated that IgG transferred by plasma neutralizes cytokines, such as interleukin (IL)-1 $\beta$ and tumor necrosis factor alpha (TNF $\alpha)$ [12].

In contrast, antibody-dependent enhancement, a mechanism in which the infection intensity increases in the presence of preexisting NAbs with poor efficacy, favors viral replication in macrophages and other cells through the interaction of these Abs with the fragment crystallizable $(\mathrm{Fc})$ and/or complement receptors [13]. If this phenomenon is suspected following CP administration, clinicians must promptly notify the health authorities and re-evaluate the safety of CP administration while taking into account the endemic coronaviruses in the region. 


\subsubsection{Fe mechanisms}

The neonatal $\mathrm{Fc}$ receptor is a vital regulator of $\operatorname{IgG}$ half-life as it prevents degradation and clearance of IgG, by a pinocytotic mechanism [14].

Intravenous immunoglobulin (IVIg) may clear autoantibodies in autoimmune conditions by shortening their lifetime [15]. Fc $\gamma$ receptors are found in almost all immune cells and their activation by IgG induces the upregulation of FC $\gamma$ RIIB, which has been associated with inhibitory effects similar to those associated with treatment efficacy for acute rejection after kidney transplantation [16]. CP infusion may help the modulation of immune responses via Fcy receptors, and merits attention in the current management of COVID-19. It may also enhance anti-inflammatory properties of dendritic cells (DCs). IVIg modulates the balance between $\mathrm{CD} 4^{+} / \mathrm{CD}^{+} \mathrm{T}$ cells and enhances proliferation and survival of regulatory $\mathrm{T}$ lymphocytes. Treatment with IVIg reduces antigenic presentation of $\mathrm{T}$ cells via the modulation and inhibition of DCs [17]. Moreover, IVIg reduces the number of Th1 and Th17 cells, and low levels of interferon gamma and TNF $\alpha$ with the increase of the levels of Th2 cytokines, such as IL-4 and IL-10 are observed after IVIg treatment [18, 19]. Furthermore, macrophages treated with IVIg showed an increased production of IL-10, with a reduction in the levels of IL-12/23p40 [20], thus, suggesting the promotion of an anti-inflammatory macrophage profile.

\section{Effects of CP transfusion in COVID-19}

On August 23, 2020, the Food and Drug Administration (FDA) granted an Emergency Use Authorization and provided guidance on the manufacture and use of CP for hospitalized patients with severe or life-threatening COVID-19. Therefore, CP is not yet approved by FDA as treatment but is regulated as an investigational product. In contrast, a National Institutes of Health guidelines panel stated that "the data are insufficient to recommend for or against the use of convalescent plasma" [21]. In addition, the American Association of Blood Banks and Infectious Diseases Society of America recommend that the use of $\mathrm{CP}$ be limited to clinical trials, as critically ill patients with COVID-19 admitted into the ICU are unlikely to benefit from administration of CP. Moreover, they recommend 
that $\mathrm{CP}$ should be transfused as early as possible in the course of the disease (preferably within three days after diagnosis) to get the best outcomes [22]. No country, including the US, has licensed CP as a treatment for COVID-19, although other countries have granted approval for use on an individual patient basis [23]. CP was mentioned in the "Diagnosis and Treatment Guidelines of COVID-19 (trial $6^{\text {th }}, 7^{\text {th }}$, and $8^{\text {th }}$ )" issued by NHC [24-26], Especially for severe and critical cases with rapid disease progression [27].

To the best of our knowledge, eight randomized controlled trials (RCTs) on the role of CP in COVID-19 management have been reported [28-34]. Six of them revealed no statistically significant differences regarding mortality, disease progression, and length of hospital admission, between patients treated with CP and the control groups. Agarwal et al. carried out the PLACID RCT, which included 464 hospitalized adults who were randomly allocated to receive either two doses of $200 \mathrm{~mL}$ $\mathrm{CP}$, with a median NAb titer of 1:40, in addition to standard care or being control. The CP group did not show any improvement in the primary outcomes (progression to severe disease or fatality at 28 days) compared to control standard care. Interestingly, NAbs were detected in $80 \%$ of the participants at the beginning of one study [29].

In agreement, two RCTs from Spain and Netherlands, terminated their studies prematurely as $50 \%$ and $79 \%$ of patients had NAb titers close to that of $\mathrm{CP}$ donors, respectively; therefore, no additional benefit would be gained from CP transfusion. No differences were noticed in fatality, duration of hospital stay, or illness severity at day $15[30,31]$. The largest worldwide RCTs, Randomized Evaluation of COVID-19 Therapy (RECOVERY; ISRCTN50189673) and Randomized, Embedded, Multifactorial Adaptive Platform Trial for Community-Acquired Pneumonia trials, by Oxford University, continuously evaluate patients with COVID-19. Regarding the CP arm, data on patients randomized to CP vs. standard care were evaluated since May 2020 till 15th of January 2021 and the preliminary analysis showed no significant difference in 28-day mortality. However, Horby et al. concluded that although the preliminary results were negative, follow-up of patients is ongoing and final results will be published in due course [28]. 
In contrast, an RCT by Rasheed et al. indicated that $\mathrm{CP}$ administration resulted in reduced illness duration and time to recovery and lower fatality rates [33]. In addition, Abolghasemi et al., found significant improvement in clinical outcomes after CP treatment, particularly, regarding hospital stay and the need for invasive ventilation. It also reduced mortality rate, but this was not statistically significant [35]. Furthermore, Libster and colleagues conducted a double-blind RCT and concluded that early transfusion of high-titer CP [IgG titer greater than 1:1,000 against SARS-CoV-2 spike (S) protein] to mildly infected older adults reduced the progression of disease in a dose-dependent manner[34]. These results are similar to the results reported by Salazar et al. who found that patients transfused within $72 \mathrm{~h}$ of hospital admission showed reduced 28-day mortality rate compared to the control group (non-transfused patients) [36].

The largest CP study, the Expanded Access Program in USA, was designed to evaluate safety. Authors documented 7- and 30-day fatality rates in 35,322 severe to critical hospitalized adult patients. Overall data suggested that CP reduced mortality; this decreased fatality had linear relationships with earlier time to transfusion (within 3 days of diagnosis) and $\mathrm{CP}$ with high Ab titer [37]. Two metaanalyses have declared uncertainty on the benefits of CP for people admitted to hospital with COVID19 [38, 39]. A recent systematic review of RCTs showed no statistically significant differences between the $\mathrm{CP}$ group and controls regarding reducing fatality and improving clinical outcomes. However, some non-RCTs and case series showed that CP may help select patients to improve clinical symptoms, neutralize and clear the virus, and decrease mortality, especially when administered early (within 10 days of illness). However, high-quality RCTs are needed to provide more evidence on the optimal administration time, Ab titers, and doses for the optimal effective use of CP [40].

\section{Donor eligibility, recruitment, and testing}

Recruitment: This was performed using national registries of patients with COVID-19, at least 14 days after complete recovery [41]. The chosen timeframe ( $\geq 14$ days) could favor NAb interactions because of increased affinity [42]. 


\section{Testing:}

1- Serological testing: Anti-SARS-CoV-2 tests provide evidence of resolved infections.

- Ab Type: Although there is uncertainty on whether total SARS-CoV-2 Abs or subclasses (e.g., IgM, IgG, or IgA) are the optimal target to measure [8], it is strongly recommended to measure NAbs.

- Ab titer: The anti-SARS-CoV-2 IgG titer should be assessed; adequate level of antiSARS-CoV-2- NAb titer is an important prerequisite, and the recommended NAb titer cut-off should be at least $\geq 1: 160$ and optimally be greater than 1:320 [43]. This corresponds to a receptor-binding domain IgG titer $\geq 1: 1,350$ [36]. A titer of 1:80 may be considered acceptable if an alternative matched unit is not available [43]. In a series of 176 patients with COVID-19, a high Ab titer (as assessed by enzyme-linked immunosorbent assay between days 22-39 after onset) was independently associated with a worse clinical classification [44]. In addition, virus inactivation methods should be strictly applied before using plasma [41].

Donor eligibility: Donor selection criteria vary according to different protocols adopted in each country.

\section{a) EU criteria:}

1. A prior diagnosis of COVID-19 based on a laboratory test or a clear history of COVID-19 symptoms where testing was not available

2. At least 14 days should have passed since full recovery or at least 14 days after laboratory evidence for viral ribonucleic acid clearance from the upper respiratory tract

3. Donors without a history of blood transfusion and female donors who have never been pregnant or are tested and found negative for anti-HLA/HPA/HNA antibodies using a validated assay

4. Informed consent

5. Aged between 18 and 65 years old 
6. Weight not less than $50 \mathrm{~kg}$

7. Standard donor criteria for blood or plasma donation must be met.

b) FDA criteria

1. Evidence of COVID-19 documented by a laboratory test either by a diagnostic test at the time of illness or a positive serological test for SARS-CoV-2 antibodies after recovery

2. Complete resolution of symptoms at least 14 days before the donation

3. Male donors, or female donors who have not been pregnant, or female donors who have been tested since their most recent pregnancy and results interpreted as negative for HLA antibodies

4. SARS-CoV-2 NAb titers, if available

o When measurement of NAb titers is available, we recommend NAb titers of at least 1:160. A titer of 1:80 may be considered acceptable if an alternative matched unit is not available.

\section{Collection, processing, and storage}

Plasma donation is ideally by plasmapheresis, as the volume of collected plasma can be adjusted based on gender, height, and weight and is approximately $400-800 \mathrm{~mL}$ of plasma from a single apheresis donation. Preferably, the collected plasma should undergo pathogen reduction treatment before being split and frozen as $2-3$ separate units (e.g., $3 \times 200 \mathrm{~mL})$ within $24 \mathrm{~h}$ of collection. Where that is not possible, (e.g., donor does not wish to undergo apheresis or logistic limitation), whole blood can also be collected. However, plasma separation from the blood has drawbacks, such as the low plasma volume collected and the inability to repeat donations in short time intervals as redonation could take place every 2 weeks by plasmapheresis but every 3 months if obtained by whole blood fractionation. Importantly, plasma is screened for human immunodeficiency virus, hepatitis B virus, hepatitis $\mathrm{C}$ virus, syphilis, human T-cell lymphotropic virus 1 and 2, and Trypanosoma cruzi (if living in an endemic area) preferred by NAT. Other protocols suggest the inactivation of pathogens with riboflavin or psoralen plus exposure to ultraviolet light to improve safety of $\mathrm{CP}[8,45]$. 


\section{Patient eligibility}

Reported studies and trials to date have mostly concentrated on hospitalized adults, including severe and critically ill patients. As the main function of $\mathrm{CP}$ is providing NAbs for viral neutralization, earlier infusion (pre-hospital in mild and moderate cases or as prophylaxis) may be of benefit and reduce disease progression, and many studies (e.g. NCT04323800 and NCT04438057) with this research focus are underway [46].

Eligibility criteria used for the National Expanded Access Treatment Protocol by FDA Center for Biologics Evaluation and Research, May 2020:

1. Laboratory confirmed COVID-19 with severe or immediately life-threatening COVID-19

2. Informed consent provided by the patient or healthcare proxy

Most importantly, the uncertainty about the efficacy of CP treating people with COVID-19 should be communicated to potential recipients to avoid fostering unfounded expectations.

\section{Dose and rate of $\mathrm{CP}$ infusion}

There is no standard transfusion dose data for $\mathrm{CP}$ administration. In different studies, CP administration ranges between $200-500 \mathrm{~mL}$ in single or double doses and is determined by the clinician based on clinical status, patient weight, and Ab titers. Currently, the recommendation is to administer $3 \mathrm{~mL} / \mathrm{kg}$ per dose in two days [8], or more accurately depending on Ab titer, it would be $3.125 \mathrm{~mL} / \mathrm{kg}$ of $\mathrm{CP}$ with a titer of $>1: 64$ would provide an equivalent $\mathrm{Ab}$ level to one-quarter of 5 $\mathrm{mL} / \mathrm{kg}$ CP with a titer of $1: 160$ or greater (e.g., $\sim 80 \mathrm{~kg}$ patient, $3.125 \mathrm{~mL} / \mathrm{kg} \times 80 \mathrm{~kg}=250 \mathrm{~mL}>1: 64$ ). Slow infusion, with a recommended speed of $100 \mathrm{~mL} / \mathrm{h}$, but not more than $200 \mathrm{~mL} / \mathrm{h}$, and close monitoring to identify and treat circulatory overload occurrence or other transfusion-related immediate side-effects must be observed. If any adverse reaction occurs, the infusion rate is slowed, suspended, or terminated [42].

\section{Timing of CP infusion}


One may hypothesize that early administration of CP containing polyclonal NAbs may inhibit viral entry and replication and consequently blunt an early pro-inflammatory pathogenic endogenous $\mathrm{Ab}$ response [47]. Therefore, it has been suggested that $\mathrm{CP}$ be administered early in the course of the disease in patients at high risk of subsequent deterioration (i.e., age above 70 or dependence on oxygen with a baseline oxygen saturation of less than 94\%) [42].

Based on the most recent data available $[44,48]$, treatment should be initiated no later than day 5. It was suggested that transfusion early-on, and up to day 10 after disease onset of two plasma units of $200-250 \mathrm{~mL}$ each in patients weighing between 50 and $80 \mathrm{~kg}$ (a volume that may be adjusted for patients weighing outside this range) is appropriate. A repeat infusion of 2 units $24-48 \mathrm{~h}$ later may be considered after verifying adequate tolerance in a first group of treated patients [42]. According to the physiopathology of COVID-19, patients with severe disease should be privileged over critical ones to reduce mortality and improve outcomes.

\section{Safety of CP and potential adverse events}

The usual plasma transfusion reaction in addition to $\mathrm{CP}$-specific concerns mainly include antibody-dependent enhancement of infection in addition to thromboembolic events [49, 50]. Treatment of CP with pathogen inactivation and reduction technologies may further minimize the risk of virus transfusion $[51,52]$.

\section{CONCLUSION}

To date, despite the large number of studies and trials, uncertainty regarding the effectiveness and safety of CP still exists. However, collective evidence indicates two cardinal players determine the effectiveness of $\mathrm{CP}$ use: time of infusion and quality of CP. Early administration of $\mathrm{CP}$ with high neutralizing anti- SARS-CoV-2 anti-spike IgG titer is hypothesized to be effective in improving clinical outcomes, prevent disease progression, decrease the length of hospital stay, and reduce mortality rate. Hence, it is assumed better to be given in moderate to severe non-ventilated patients with COVID-19 (before the cytokine storm and end organ damage) than in critically ill patients. 
However, more reliable, high quality, well-designed and well-controlled double-blinded randomized international and multicenter collaborative trials are still needed to establish CP safety, effectiveness, detailed optimal product criteria as well as reliable guidelines for best clinical use to provide the maximum benefit and avoid harm.

\section{Authors' Disclosure of Potential Conflict of Interest}

No potential conflicts of interest relevant to this article were reported. 


\section{REFERENCES}

1. WHO Blood Regulators Network. Position paper on use of convalescent plasma, serum or immune globulin concentrates as an element in response to an emerging virus. Geneva, Switzerland: WHO Blood Regulators Network, 2017. (Accessed August 3, 2021, at https:// www.who.int/bloodproducts/brn/2017_BRN_PositionPaper_ConvalescentPlasma .pdf?ua=1).

2. Lai CC, Shih TP, Ko WC, Tang HJ, Hsueh PR. Severe acute respiratory syndrome coronavirus 2 (SARS-CoV-2) and coronavirus disease-2019 (COVID-19): the epidemic and the challenges. Int J Antimicrob Agents 2020;55:105924.

3. Wang M, Cao R, Zhang L, et al. Remdesivir and chloroquine effectively inhibit the recently emerged novel coronavirus (2019-nCoV) in vitro. Cell Res 2020;30:269-71.

4. Marano G, Vaglio S, Pupella S, et al. Convalescent plasma: new evidence for an old therapeutic tool? Blood Transfus 2016;14:152-7.

5. Rajendran K, Krishnasamy N, Rangarajan J, Rathinam J, Natarajan M, Ramachandran A. Convalescent plasma transfusion for the treatment of COVID-19: systematic review. J Med Virol 2020;92:1475-83.

6. Du L, He Y, Zhou Y, Liu S, Zheng BJ, Jiang S. The spike protein of SARS-CoV--a target for vaccine and therapeutic development. Nat Rev Microbiol 2009;7:226-36.

7. Wu F, Wang A, Liu M, et al. Neutralizing antibody responses to SARS-CoV-2 in a COVID19 recovered patient cohort and their implications. medRxiv 2020:20047365.

8. Bloch EM, Shoham S, Casadevall A, et al. Deployment of convalescent plasma for the prevention and treatment of COVID-19. J Clin Invest 2020;130:2757-65.

9. Hsueh PR, Huang LM, Chen PJ, Kao CL, Yang PC. Chronological evolution of IgM, IgA, $\mathrm{IgG}$ and neutralisation antibodies after infection with SARS-associated coronavirus. Clin Microbiol Infect 2004;10:1062-6.

10. Rokni M, Ghasemi V, Tavakoli Z. Immune responses and pathogenesis of SARS-CoV-2 during an outbreak in Iran: comparison with SARS and MERS. Rev Med Virol 2020;30:e2107. 
11. Zhang Y, Xiao M, Zhang S, et al. Coagulopathy and antiphospholipid antibodies in patients with Covid-19. N Engl J Med 2020;382:e38.

12. Abe Y, Horiuchi A, Miyake M, Kimura S. Anti-cytokine nature of natural human immunoglobulin: one possible mechanism of the clinical effect of intravenous immunoglobulin therapy. Immunol Rev 1994;139:5-19.

13. Antibody-dependent enhancement of viral infections. In: Kulkarni R, ed. Dynamics of immune activation in viral diseases. Singapore: Springer, 2019:9-41.

14. Chaigne B, Mouthon L. Mechanisms of action of intravenous immunoglobulin. Transfus Apher Sci 2017;56:45-9.

15. Akilesh S, Petkova S, Sproule TJ, Shaffer DJ, Christianson GJ, Roopenian D. The MHC class I-like Fc receptor promotes humorally mediated autoimmune disease. J Clin Invest 2004;113:1328-33. 16. Jin J, Gong J, Lin B, Li Y, He Q. FcyRIIb expression on B cells is associated with treatment efficacy for acute rejection after kidney transplantation. Mol Immunol 2017;85:283-92.

17. Aubin E, Lemieux R, Bazin R. Indirect inhibition of in vivo and in vitro T-cell responses by intravenous immunoglobulins due to impaired antigen presentation. Blood 2010;115:1727-34.

18. Ahmadi M, Abdolmohammadi-Vahid S, Ghaebi M, et al. Effect of intravenous immunoglobulin on Th1 and Th2 lymphocytes and improvement of pregnancy outcome in recurrent pregnancy loss (RPL). Biomed Pharmacother 2017;92:1095-102.

19. McGonagle D, Sharif K, O'Regan A, Bridgewood C. The role of cytokines including interleukin-6 in COVID-19 induced pneumonia and macrophage activation syndrome-like disease. Autoimmun Rev 2020;19:102537.

20. Kozicky LK, Zhao ZY, Menzies SC, et al. Intravenous immunoglobulin skews macrophages to an anti-inflammatory, IL-10-producing activation state. J Leukoc Biol 2015;98:983-94.

21. Katz LM. (A Little) Clarity on convalescent plasma for Covid-19. N Engl J Med 2021;384:666-8.

22. Infectious Diseases Society of America. Clarifying the emergency use authorization framework for COVID-19 convalescent plasma: considerations for clinicians prepared jointly by the 
Infectious Diseases Society of America and AABB. Arlington, VA: Infectious Diseases Society of America, 2020. (Accessed August 3, 2021, at https://www.idsociety.org/globalassets/covid-19-realtime-learning-network/therapeutics-and-interventions/convalescent-plasma/aabb-idsa-convalescentplasma-eua--final.pdf).

23. Estcourt LJ, Roberts DJ. Convalescent plasma for covid-19. BMJ 2020;370:m3516.

24. National Health Commission of the People's Republic of China. Protocol on Prevention and Control of COVID-19. 6th ed. Beijing, China: National Health Commission of the People's Republic of China, 2020.

25. National Health Commission of the People's Republic of China. Diagnosis and Treatment Protocol for COVID-19. Trial Version 7. Beijing, China: National Health Commission of the People's Republic of China, 2020.

26. National Health Commission of the People's Republic of China. Full text: Diagnosis and Treatment Protocol for COVID-19 Patients. Tentative 8th ed. Beijing, China: National Health Commission of the People's Republic of China, 2020.

27. Chen L, Xiong J, Bao L, Shi Y. Convalescent plasma as a potential therapy for COVID-19. Lancet Infect Dis 2020;20:398-400.

28. University of Oxford. Randomised evaluation of COVID-19 therapy (recovery). Oxford, UK; University of Oxford, 2020. (Accessed August 3, 2021, at https://clinicaltrials.gov/ct2/show/record/NCT04381936).

29. Agarwal A, Mukherjee A, Kumar G, et al. Convalescent plasma in the management of moderate covid-19 in adults in India: open label phase II multicentre randomised controlled trial (PLACID Trial). BMJ 2020;371:m3939.

30. Avendaño-Solà C, Ramos-Martínez A, Muñez-Rubio E, et al. Convalescent plasma for COVID-19: a multicenter, randomized clinical trial. medRxiv 2020:20182444.

31. Gharbharan A, Jordans CEC, Geurtsvankessel C, et al. Convalescent plasma for COVID-19. A randomized clinical trial. medRxiv 2020:20139857. 
32. Li L, Zhang W, Hu Y, et al. Effect of convalescent plasma therapy on time to clinical improvement in patients with severe and life-threatening COVID-19: a randomized clinical trial. JAMA 2020;324:460-70.

33. Rasheed AM, Fatak DF, Hashim HA, et al. The therapeutic potential of convalescent plasma therapy on treating critically-ill COVID-19 patients residing in respiratory care units in hospitals in Baghdad, Iraq. Infez Med 2020;28:357-66.

34. Libster R, Pérez Marc G, Wappner D, et al. Early high-titer plasma therapy to prevent severe Covid-19 in older adults. N Engl J Med 2021;384:610-8.

35. Abolghasemi H, Eshghi P, Cheraghali AM, et al. Clinical efficacy of convalescent plasma for treatment of COVID-19 infections: results of a multicenter clinical study. Transfus Apher Sci 2020;59:102875.

36. Salazar E, Christensen PA, Graviss EA, et al. Treatment of coronavirus disease 2019 patients with convalescent plasma reveals a signal of significantly decreased mortality. Am J Pathol 2020;190:2290-303.

37. Joyner MJ, Wright RS, Fairweather D, et al. Early safety indicators of COVID-19 convalescent plasma in 5000 Patients. J Clin Invest 2020;130:4791-7.

38. Piechotta V, Chai KL, Valk SJ, et al. Convalescent plasma or hyperimmune immunoglobulin for people with COVID-19: a living systematic review. Cochrane Database Syst Rev 2020;7:CD013600.

39. Chai KL, Valk SJ, Piechotta V, et al. Convalescent plasma or hyperimmune immunoglobulin for people with COVID-19: a living systematic review. Cochrane Database Syst Rev 2020;10:CD013600.

40. Wang Y, Huo P, Dai R, et al. Convalescent plasma may be a possible treatment for COVID19: a systematic review. Int Immunopharmacol 2021;91:107262.

41. Selvi V. Convalescent plasma: a challenging tool to treat COVID-19 patients-a lesson from the past and new perspectives. Biomed Res Int 2020;2020:2606058. 
42. Tiberghien P, de Lamballerie X, Morel P, Gallian P, Lacombe K, Yazdanpanah Y. Collecting and evaluating convalescent plasma for COVID-19 treatment: why and how? Vox Sang 2020;115:488-94.

43. Zhao J, Yuan Q, Wang H, et al. Antibody responses to SARS-CoV-2 in patients with novel coronavirus disease 2019. Clin Infect Dis 2020;71:2027-34.

44. Bello-López JM, Delgado-Balbuena L, Rojas-Huidobro D, Rojo-Medina J. Treatment of platelet concentrates and plasma with riboflavin and UV light: impact in bacterial reduction. Transfus Clin Biol 2018;25:197-203.

45. Wood EM, Estcourt LJ, McQuilten ZK. How should we use convalescent plasma therapies for the management of COVID-19? Blood 2021;137:1573-81.

46. Walls AC, Park YJ, Tortorici MA, Wall A, McGuire AT, Veesler D. Structure, function, and antigenicity of the SARS-CoV-2 spike glycoprotein. Cell 2020;181:281-92.e6.

47. Wölfel R, Corman VM, Guggemos W, et al. Virological assessment of hospitalized cases of coronavirus disease 2019. medRxiv 2020:20030502.

48. Psaltopoulou T, Sergentanis TN, Pappa V, et al. The emerging role of convalescent plasma in the treatment of COVID-19. Hemasphere 2020;4:e409.

49. Shankar-Hari M, Estcourt L, Harvala H, Roberts D, Menon DK; United Kingdom SARSCoV-2 Convalescent Plasma Evaluation (SCoPE) Consortium. Convalescent plasma to treat critically ill patients with COVID-19: framing the need for randomised clinical trials. Crit Care 2020;24:449.

50. Sullivan HC, Roback JD. Convalescent plasma: therapeutic hope or hopeless strategy in the SARS-CoV-2 pandemic. Transfus Med Rev 2020;34:145-50.

51. Dzik S. COVID-19 convalescent plasma: now is the time for better science. Transfus Med Rev 2020;34:141-4.

52. Rojas M, Rodríguez Y, Monsalve DM, et al. Convalescent plasma in Covid-19: possible mechanisms of action. Autoimmun Rev 2020;19:102554.

Figure 1 
A. CONVALESCENT PLASMA

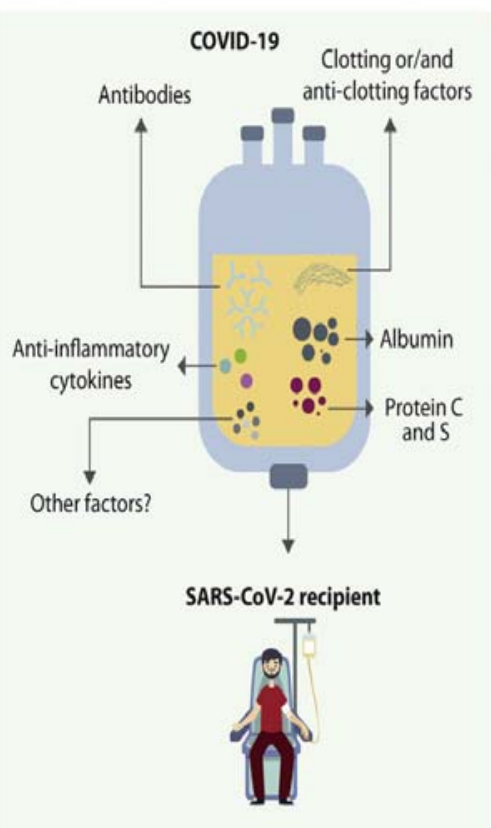

B. ANTIVIRAL EFFECTS

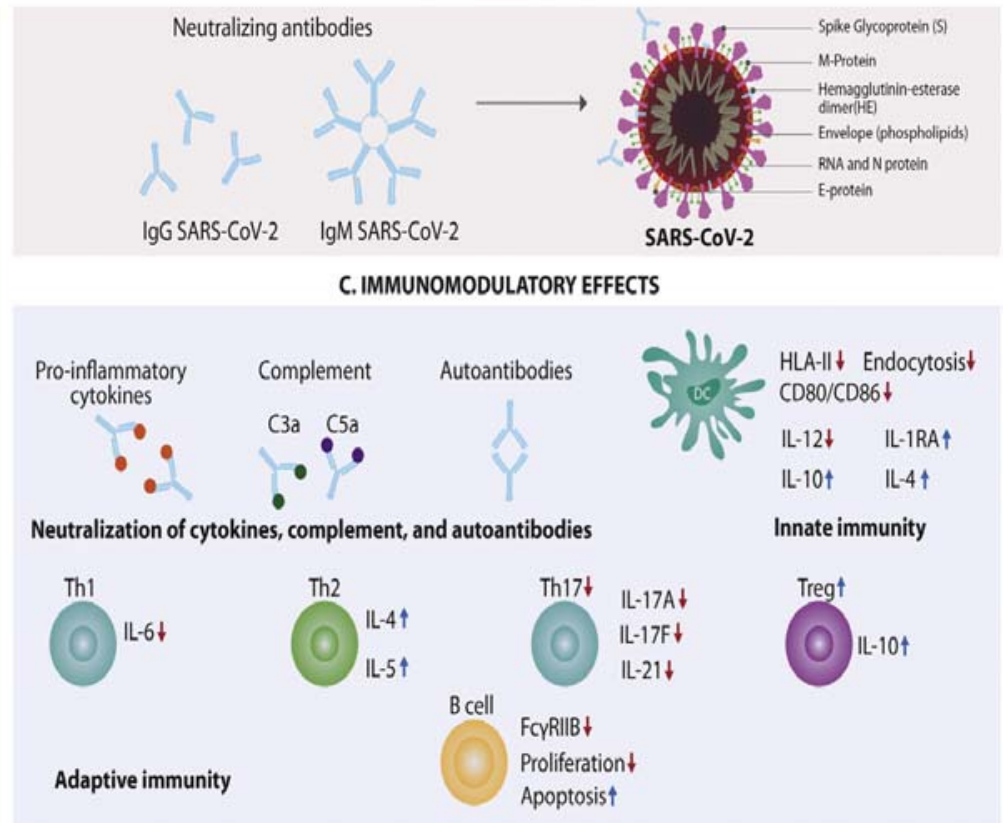

Fig. 1. Schematic representation of convalescent plasma (CP) components and its mechanisms of action. (A) Main convalescent plasma components. (B) Antiviral effects of neutralizing antibodies (NAbs). Immunoglobulin (Ig)G and IgM are the main isotypes, although IgA may be also important, particularly in mucosal viral infections. Other non-NAbs may exert a protective effect. The humoral immune response is mainly directed towards the spike (S) protein. (C) Anti-inflammatory effects of CP include network of autoantibodies and control of an overactive immune system (i.e., cytokine storm, Th1/Th17 ratio, complement activation, and regulation of a hypercoagulable state) [52].

Abbreviations: E, envelope; M, membrane; $\mathrm{N}$, nucleoprotein. 